\title{
Dieta de murciélagos nectarívoros del Parque Nacional Cerros de Amotape, Tumbes
}

\section{Diet of nectarivorous bats from the National Park Cerros de Amotape, Tumbes}

\author{
Edith Arias ${ }^{1}$, Richard Cadenillas ${ }^{1}$ y Víctor Pacheco ${ }^{1,2}$
}

1 Museo de Historia Natural, Uni(1) versidad Nacional Mayor de San Mer, Apartado 14-0434, LimaPerú. Email Edith Arias: ediari_char@yahoo.es

2 Facultad de Ciencias Biológicas, Universidad Nacional Mayor de San Marcos.
Presentado: $\quad 19 / 09 / 2009$

\section{Resumen}

En el Perú se reporta la presencia de 18 especies de murciélagos nectarívoros, sin embargo se cuenta con poca información sobre la dieta de estas especies. En este estudio se reporta por primera vez la dieta de los nectarívoros Glossophaga soricina, Lonchophylla hesperia y Anoura geoffroyi en el bosque seco ecuatorial y del bosque tropical del Pacífico del Parque Nacional Cerros de Amotape, Tumbes. Analizamos 21 contenidos gastrointestinales e identificamos ocho morfotipos de polen pertenecientes a las familias Bombacaceae, Cactaceae, Fabaceae, Solanaceae, Rubiaceae, Myrtaceae, Malvaceae y Rosaceae. Encontramos evidencia del síndrome de quiropterofilia en Bombacaceae, Cactaceae, Fabaceae, Solanaceae y Rubiaceae. Observamos que A. geoffroyi consume polen de Ceiba trichistandra, Solanaceae y Rubiacea; G. soricina consume de Abutilon reflexum, Armathocereus cartwrightianus, C. trichistandra y Rubiaceae; y L. hesperia de A. cartwrightianus, Eriobotrya japonica, Fabaceae y Psidium sp.; sugiriendo una dieta generalista en estas especies. Los murciélagos $G$. soricina y $A$. geoffroyi comparten el consumo del ceibo $C$. trichistandra y de la Rubiaceae, mientras que $G$. soricina comparte con $L$. hesperia el consumo del cactus $A$. cartwrightianus. Los otros morfotipos de polen no fueron compartidos entre murciélagos. Se encuentra además que el ceibo $C$. trichistandra fue la especie más consumida, especialmente por G. soricina.

Palabras clave: Nectarívoros, quiropterofilia, Chiroptera, polinizadores, áreas protegidas.

\section{Abstract}

In Peru 18 species of nectarivorous bats are reported, however little information about their diet is available. This study is the first report about pollen consumption of the nectar-feeding bat species Glossophaga soricina, Lonchophylla hesperia, and Anoura geoffroyi in the dry forest and the Pacific Tropical rainforest of the National Park Cerros de Amotape, Tumbes. We analyzed 21 stomach contents and identified eigth pollen morphotypes belonging to the families Bombacaceae, Cactaceae, Fabaceae, Solanaceae, Rubiaceae, Myrtaceae, Malvaceae, and Rosaceae; and found floral evidences of the chiropterophilous syndrome in Bombacaceae, Cactaceae, Fabaceae, Solanaceae, and Rubiaceae. We found that $A$. geoffroyi consumed pollen of Ceiba trichistandra, Solanaceae, and Rubiaceae; $G$ soricina consumed of Abutilon reflexum, Armathocereus cartwrightianus, $C$. trichistandra, and Rubiaceae; and L. hesperia of A. cartwrightianus, Eriobotrya japonica, Fabaceae, and Psidium sp.; suggesting that these bat species have a generalist diet. The bats $G$. soricina and $A$. geoffroyi shared the consumption of the ceiba $C$. trichistandra and the Rubiaceae, while G. soricina and $L$. hesperia shared the consumption of the cactus $A$. cartwrightianus. The other five morphotypes were not shared. In addition, we found that the ceiba $C$. trichistandra was the species most consumed by bats, specially G. soricina.

Keywords: Nectarivorous, chiropterophilous, Chiroptera, pollinators, Protected Areas.

\section{Introducción}

En el Perú se reportan 18 especies de murciélagos nectarívoros (Pacheco et al. 2009), de las cuales Anoura geoffroyi, Glossophaga soricina, Lonchophylla hesperia y Choeroniscus minor se encuentran presentes en el bosque seco ecuatorial y del bosque tropical del Pacífico del Perú (Pacheco et al. 2007). Estudios sobre la dieta de especies nectarívoras y su función en la polinización no son conocidos, en particular en los bosques del norte del Perú. Sin embargo han sido realizados estudios en otras partes del Neotrópico, especialmente en G.soricina y A. geoffroyi (Sánchez y Álvarez 2000, Sazima et al. 1999, Quesada et al. 2003, 2004, Helversen 2002, Muchhala y Jarrín-V 2002).

Glossophaga soricina es considerada un nectarívoro oportunista, consumidor de plantas con o sin el síndrome de quiropterofilia (Sánchez y Álvarez 2000); y con una alta preferencia por la familia Bombacaceae en los bosques secos de México y Costa Rica (Lobo et al. 2003, Quesada et al. 2003, 2004).

Por otro lado, $A$. geoffroyi ha sido documentado como consumidor y transportador de polen de plantas quiropterofílicas en hábitats de la selva baja caducifolia de México y la Cuenca Amazónica de Brasil (Sazima et al. 1999, Caballero-Martínez et al. 2009). Esta misma situación ha sido observada en la vertiente occidental de los Andes del Ecuador donde $A$. geoffroyi además de su preferencia hacia plantas con el síndrome de quiropterofilia, actúa como polinizador de ellas (Muchhala y Jarrín-V. 2002).

Choeroniscus minor cuenta con poca información sobre su dieta excepto por el trabajo de Delaval et al. (2005) quienes reportan la polinización de una Fabaceae por esta especie en la Guyana Francesa. Finalmente, no se conocen trabajos sobre la dieta de L. hesperia.

Este estudio presenta el primer análisis cualitativo y cuantitativo del polen consumido por los murciélagos nectarívoros en el bosque seco y tropical del Pacífico de Tumbes y representa uno de los primeros en su tipo para el Perú.

\section{Material y métodos}

Se analizó el contenido gastrointestinal de 21 especímenes de Glossophaga soricina (16 individuos), Lonchophylla hesperia (3) y Anoura geoffroyi (2), colectados entre el año 2004 y 2006, en el Parque Nacional de Cerros de Amotape, departamento de Tumbes, Perú. El 80\% de las muestras provinieron de la ecorregión de bosque seco y fueron capturadas en época húmeda, mientras que el $20 \%$ restante se colectaron en el bosque tropical del Pacífico y en época seca. Los especímenes se encuentran depositados en el Museo de Historia Natural de la Universidad Nacional Mayor de San Marcos (MUSM). 


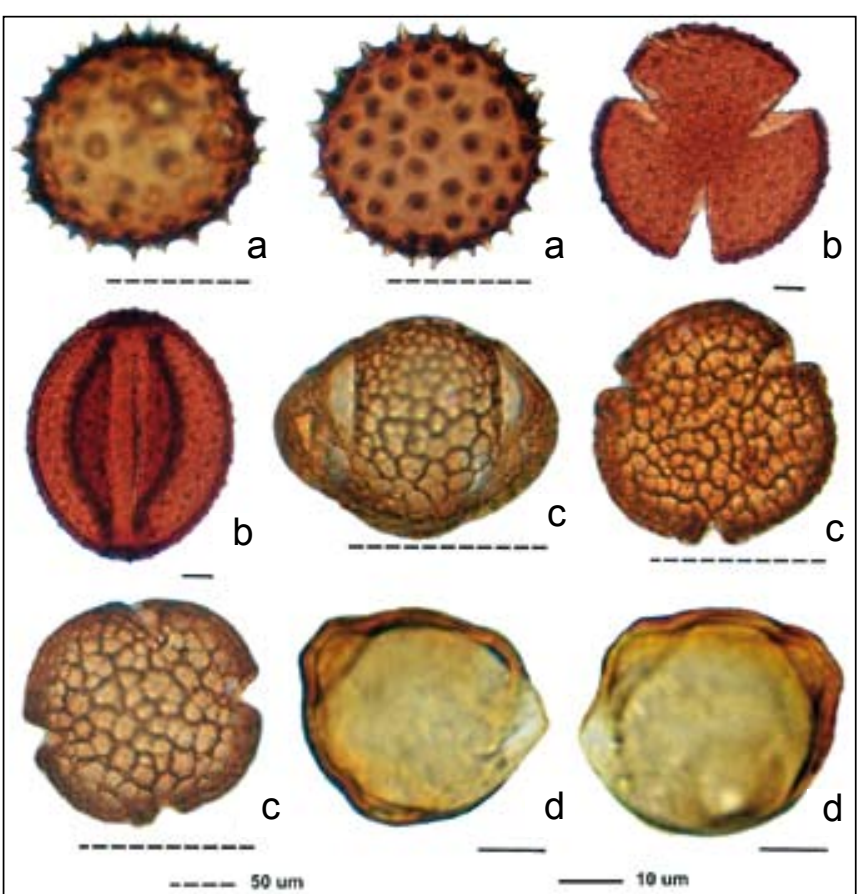

Figura 1. Granos de polen identificados a nivel de especie. (a) Abutilon reflexum, (b) Armathocereus cartwrightianus, (c) Ceiba trichistandra, (d) Eriobotrya japonica.

El contenido gastrointestinal fue sometido a un proceso de acetólisis-KOH (Álvarez y González-Quintero 1970). Las muestras de polen fueron montadas en glicerina para su posterior observación microscópica y conservación en MUSM. El polen fue identificado usando una colección referencial formada por muestras colectadas en el área de estudio y complementada con polen obtenido de plantas de los bosques del norte del Perú depositadas en el Herbario del Museo de Historia Natural de la Universidad Nacional Mayor de San Marcos. Adicionalmente, se empleó catálogos de polen y claves dicotómicas (Sáenz y Blume 1978, Erdtman 1986, Roubik y Moreno 1991). Para evaluar la importancia del polen de cada especie de planta en la dieta de $G$. soricina, $L$. hesperia y $A$. geoffroyi, se consideró su presencia en los individuos, el número total de granos de polen $(\mathrm{N})$, así como el porcentaje de los mismos.

\section{Resultados}

Se reconoció granos de polen en 16 de los 21 especímenes analizados (81\%). Anoura geoffroyi registró el 100\% de individuos con polen en su contenido gastrointestinal, mientras que $G$. soricina y $L$. hesperia registraron 81 y $67 \%$ respectivamente.

Se identificaron ocho morfotipos de polen pertenecientes a las familias Bombacaceae, Cactaceae, Fabaceae, Malvaceae, Myrtaceae, Solanaceae, Rosaceae y Rubiaceae (Tabla 1, Fig. 1 y 2). De ellas, cuatro muestras fueron identificadas hasta especie, una hasta género y tres a nivel de familia. En estas familias, cinco especies: Abutilon reflexum, Armathocereus cartwrightianus, Ceiba trichistandra, Rubiaceae y Solanaceae fueron las más abundantes ya que se encontraron en mayores cantidades; la abundancia de las demás especies de plantas fueron menores al 5\% (Tabla 1).

Se encontró que $G$. soricina consume cuatro morfotipos, el $49 \%$ de granos de polen corresponden a $A$. reflexum, el $24 \%$ a $C$. trichistandra; el $19 \%$ a $A$. cartwrightianus y el $7 \%$ a la Rubiaceae. A. geoffroyi consume tres morfotipos, el $44 \%$ de una solanácea, $36 \%$ de C. trichistandra y el $20 \%$ a una Rubiaceae. Así también se encontró que $L$. hesperia consume cuatro morfotipos, el $40 \%$ de $A$. cartwrightianus, $32 \%$ de Eriobotrya japonica; mientras que Psidium sp. y el morfotipo de la Fabaceae se encontraron en porcentajes menores al $20 \%$ cada uno. La cantidad de polen encontrada en $L$. hesperia fue menor en comparación a los otros dos nectarívoros (Tabla 1).

Glossophaga soricina y A. geoffroyi comparten el consumo de $C$. trichistandra y el morfotipo de la Rubiaceae, así también como G. soricina comparte con $L$. hesperia el consumo de $A$. cartwrightianus. Los demás morfotipos de polen no son recursos compartidos entre las especies analizadas.

De los trece individuos de $G$. soricina registrados con polen, diez individuos consumieron $C$. trichistandra, los otros tres consumieron un solo morfotipo, sea de $A$. reflexum, $A$ cartwrightianus o una Rubiaceae; mostrando así una mayor preferencia de $G$. soricina por $C$. trichistandra, a pesar de encontrarse un mayor porcentaje de polen de $A$. reflexum. Los pocos individuos analizados de $A$. geoffroyi y $L$. hesperia no permiten sugerir la preferencia de estas especies por alguna planta (Tabla 1).

\section{Discusion}

Los resultados muestran que los murciélagos nectarívoros Anoura geoffroyi, Glossophaga soricina y Lonchophylla hesperia consumen por lo menos ocho especies de plantas presentes en el Parque Nacional Cerros de Amotape. Glossophaga soricina ha sido reconocido como consumidor de plantas del género Ceiba en hábitats del bosque seco de México y Costa Rica. Kudsen (1998) reportó en los bosques secos del Ecuador la visita de un murciélago no identificado a la especie Ceiba trichistandra. Nosotros confirmamos por primera vez que $G$. soricina consume a la Bombacaceae $C$. trichistandra, así como granos de polen de la Malvaceae Abutilon reflexum, especie no quiropterofílica pero usada por estar disponible todo el día. Este resultado es consistente con la hipótesis de Sazima et al. (1999), quienes indicaron que las plantas de Abutilon, debido a su floración anual, son aprovechadas por los murciélagos como $A$. caudifer.

La cactofilia por parte de $G$. soricina, ha sido reportada por Simmons y Wetterer (2002), quienes la documentan como consumidor oportunista de cactáceas; lo cual es apoyado por nuestra observación. Al igual que Sazima et al. (1999) encontramos que $G$. soricina consume polen de una Rubiaceae; según estos autores las especies de esta familia de plantas presentan

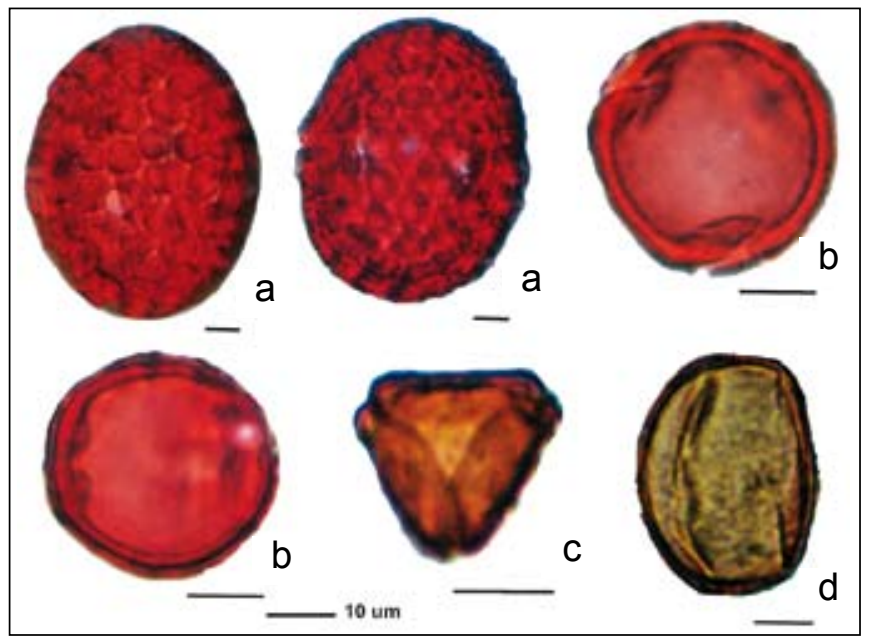

Figura 2. Granos de polen identificados a nivel de familia y género. a) Rubiaceae, b) Solanaceae, c) Psidium sp., d) Fabaceae. 
Tabla 1. Granos de polen encontrados en el contenido gastrointestinal de los murciélagos nectarívoros Anoura geoffroyi, Glossophaga soricina y Lonchophylla hesperia en el bosque seco y bosque tropical del Pacífico, Tumbes. Número de granos de polen (N), Porcentaje de granos de polen $(P)$ y Frecuencia de individuos con registros de polen $(F)$. ${ }^{*}$ Número total de individuos analizados.

\begin{tabular}{|c|c|c|c|c|c|c|c|c|c|c|c|}
\hline \multirow{2}{*}{ Familias y especies } & \multicolumn{3}{|c|}{ Anoura geoffroyi $(2)^{*}$} & \multicolumn{3}{|c|}{ Glossophaga soricina $(13)^{*}$} & \multicolumn{3}{|c|}{ Lonchophylla hesperia $(2)^{*}$} & \multicolumn{2}{|c|}{$\begin{array}{c}\text { Total de granos } \\
\text { de polen }\end{array}$} \\
\hline & $\mathrm{N}$ & $\mathrm{P}$ & $\mathrm{F}$ & $\mathrm{N}$ & $\mathrm{P}$ & $\mathrm{F}$ & $\mathrm{N}$ & $\mathrm{P}$ & $\mathrm{F}$ & $\mathrm{N}$ & $\mathrm{P}$ \\
\hline \multicolumn{12}{|l|}{ Bombacaceae } \\
\hline Ceiba trichistandra & 82 & 36 & 1 & 400 & 24 & 10 & - & - & - & 482 & 25 \\
\hline \multicolumn{12}{|l|}{ Cactaceae } \\
\hline Armatocereus cartwrightianus & - & - & - & 315 & 19 & 1 & 25 & 40 & 1 & 340 & 17,7 \\
\hline \multicolumn{12}{|l|}{ Fabaceae } \\
\hline Sp. 1 & - & - & - & - & - & - & 7 & 11 & 1 & 7 & 0,4 \\
\hline \multicolumn{12}{|l|}{ Malvaceae } \\
\hline Abutilon reflexum & - & - & - & 800 & 49 & 1 & - & - & - & 800 & 41,5 \\
\hline \multicolumn{12}{|l|}{ Myrtaceae } \\
\hline Psidium sp. & - & - & - & - & - & - & 10 & 16 & 1 & 10 & 0,5 \\
\hline \multicolumn{12}{|l|}{ Rosaceae } \\
\hline Eriobotrya japonica & - & - & - & - & - & - & 20 & 32 & 1 & 20 & 1 \\
\hline \multicolumn{12}{|l|}{ Solanaceae } \\
\hline Sp. 1 & 100 & 44 & 1 & - & - & - & - & - & - & 100 & 5,2 \\
\hline \multicolumn{12}{|l|}{ Rubiaceae } \\
\hline Sp. 1 & 46 & 20 & 1 & 121 & 7 & 1 & - & - & - & 167 & 8,7 \\
\hline Total de granos de polen & 228 & 100 & - & 1636 & 100 & - & 62 & 100 & - & 1926 & 100 \\
\hline
\end{tabular}

altos valores de néctar y azúcares que la hacen buen recurso para los nectarívoros.

La especie $A$. geoffroyi ha sido registrada como polinizador de trece familias de plantas, presentando todas estas especies el síndrome de quiropterofilia (Muchhala y Jarrín-V. 2002). Igualmente Caballero-Martínez et al. 2009, registran que este nectarívoro consume plantas que presentan este síndrome. Sazima et.al. (1999) reporta también que una especie de la familia Rubiaceae es polinizada por Anoura. Todos estos autores reportan el consumo de Bombacaceae, Solanaceae y Rubiaceae. Nosotros también reportamos el consumo de estas familias, en particular de la especie $C$. trichistandra, que es registrada por primera vez en $A$. geoffroyi.

Con respecto a la dieta de $L$. hesperia se reporta por primera vez el consumo de la cactácea $A$. cartwrightianus, una Fabaceae, la Rosaceae E. japonica y la Myrtaceae Psidium sp. Armatocereus cartwrightianus es considerada una planta quiropterofílica (Simmons y Wetterer 2002), mientras que E. japonica y Psidium sp. carecen del síndrome de quiropterofilia, de acuerdo a características mencionadas por Sazima et al. (1999) y Muchhala y Jarrín-V. (2002). Geiselman et al. (2007) registran que los frutos de E. japonica y Psidium sp. son fuente de alimento de algunas especies frugívoras, sin embargo el consumo de polen no había sido reportado. Sazima et al. 1999 y Lobo et al. 2003 registran que algunas especies de la familia Fabaceae son consumidas por nectarívoros, así también nosotros reportamos el consumo de una Fabaceae por parte L. hesperia.

Se presume que el motivo de la poca cantidad de polen encontrado en $L$. hesperia, en comparación a las otras especies nectarívoras se debe a que los especímenes fueron colectados pasadas las 2100 horas. Debido a que la digestión en murciélagos es muy rápida es posible que estos especímenes, al ser capturados, hayan ya digerido y evacuado el polen. También es posible que algunas especies no quiropterofílicas tengan poca cantidad de polen disponible.

De las ocho especies consumidas por $G$. soricina, A. geoffroyi y $L$ hesperia, cinco especies han sido previamente reportadas como familias con el síndrome de quiropterofilia: C. trichistandra (Bombacaceae), A. cartwrightianus (Cactaceae), Fabaceae, Rubiaceae y Solanaceae, dado que estas tienen flores con colores inconspicuos, antítesis nocturna, gran cantidad de polen y producción de néctar por la noche (Knudsen y Klitgaard 1998; Sazima et al. 1999; Muchhala y Jarrín-V 2002; Simmons y Wetterer 2002). Las otras tres especies han sido consideradas como fuente de recurso de nectarívoros sin presentar el síndrome de quiropterofilia (Sazima et al.1999; Geiselman et al. 2007).

Es interesante saber que $A$. geoffroyi comparte el uso de $C$. trichistandra con $G$. soricina, debido a que los especímenes de A. geoffroyi fueron registrados en época seca y los de G. soricina en época húmeda. Este hecho nos demuestra la disponibilidad de C. trichistandra en ambas estaciones; favoreciendo a estas especies en la obtención de sus recursos alimenticios.

Simmons y Wetterer (2002), indicaron que los murciélagos cactófilos tienden a tener cráneos y antebrazos más largos así como mayor peso corporal, características que facilitan el acceso al néctar. Es así que $L$. hesperia se ajusta a estas características comparándola con $G$. soricina haciéndonos predecir una posible competencia entre estas dos especies que comparten el consumo del cactus $A$. cartwrightianus.

La preferencia del ceibo $C$. trichistandra por parte de $G$. soricina es evidente, la mayoría de individuos optaron por este recurso debido a la condición quiropterofílica que presenta, sumado a la oferta de sus recursos (Knudsen y Klitgaard 1998). Especies de la familia Bombacaceae han sido relacionadas con los murciélagos nectarívoros, debido a que estos actúan como verdaderos polinizadores de árboles neotropicales, en especial del género Ceiba (Lobo et al. 2003; Quesada et al. 2003, 2004) No obstante la preferencia de $G$. soricina por $C$. trichistandra, este nectarívoro reporta un mayor consumo de polen de $A$. reflexum, debido sin duda a que esta Malvaceae presenta una mayor producción de polen.

Anoura geoffroyi y Lonchophylla hesperia no evidenciaron preferencias por ninguna especie, resultado que podría estar influenciado por la poca cantidad de individuos analizados. 
Nuestros resultados son uno de los primeros reportes sobre el consumo de polen por murciélagos nectarívoros en el Perú, en plantas con o sin el síndrome de quiropterofilia; añadiéndose a los estudios pioneros de Sahley (1996), quien evidenció por primera vez para el Perú un caso de quiropterofilia de la especie Weberbauerocereus weberbaueri (Cactaceae) por el murciélago nectarívoro Platalina genovensium.

En base al hecho de que $A$. geoffroyi, G. soricina, y L. hesperia, visitan las plantas para obtener polen se propone que estas especies de murciélagos actúan como polinizadores de las plantas mencionadas. Sin embargo para sustentar este argumento se requerirá un estudio que demuestre el traspaso del polen de los estambres hacia el estigma de las flores; y la consecuencia de la polinización en el suceso reproductivo de las plantas (Fleming y Sosa 1994).

Mucho queda aún por conocer de las 18 especies de murciélagos nectarívoros presentes en el Perú, el consumo de distintas especies de plantas sugieren una dieta generalista en Anoura geoffroyi, Glossophaga soricina y Lonchophylla hesperia; reforzando los estudios que encuentran una dieta generalista en la mayoría de murciélagos nectarívoros (Lemke 1985, Muchhala y Jarrín V. 2002). Este tipo de dieta es aparentemente ventajoso ya que estos murciélagos pueden satisfacer sus necesidades alimenticias con una mayor variedad de plantas y en diferentes épocas del año (Lemke 1985, Muchhala y Jarrín V. 2002).

\section{Agradecimientos}

La presente investigación recibió el apoyo del Instituto de Ciencias Biológicas "Antonio Raimondi” y el Consejo Superior de Investigaciones-UNMSM (Proyectos 041001081, 051001011 y 061001021), una beca María Koepcke de APECO -Conservación Internacional Perú (proyecto 16-2006) y Bat Conservation Internacional (BCI). Nuestro agradecimiento al Herbario San Marcos (USM) por permitir la revisión de plantas y toma de muestras. También a Liz Huamaní, Sandra Velazco, Úrsula Fajardo y Edith Salas, integrantes del departamento de Mastozoología del Museo de Historia Natural de la UNMSM, por su valiosa ayuda en las colectas. Y otro especial a Sandra Velazco por sus observaciones y revisión del manuscrito.

\section{Literatura citada}

Álvarez T. \& González-Quintero. 1970. Análisis polínico del contenido gástrico de murciélagos Glossophagine de México. Anales de la Escuela Nacional de Ciencias Biológicas, México, 18: 137-165.

Caballero-Martínez L. A. \& I. Rivas, L. Aguilera. 2009. Hábitos alimentarios de Anoura geoffroyi (Chiroptera: Phyllostomidae) en Ixtapan del Oro, Estado de México. Acta Zoológica Mexicana (n.s) 25(1): 161-175.

Delaval M., M. Henry \& M. Charles-Dominique. 2005. Interspecific competition and niche partitioning: example of a Neotropical rainforest bat community. Rev. Ecol. (Terre Vie) 60: 149-165.

Erdtman G. 1986. Pollen morphology and plant taxonomy: Angiosperms (An introduction to Palynology). E. J. Brill, Leiden, The Netherlands. 553 pp.

Fleming T.H. \& V.J. Sosa. 1994. Effects of nectarivorous and frugivorous mammals on reproductive success of plants. Journal of Mammalogy 75(4):845-851.
Geiselman C. K., S. A. Mori, F. Blanchard. 2007. (en línea) Database of Neotropical bat/Plant interactions. http://www.nybg.org/ botany $/$ tlobova $/$ mori/batsplants/database/dbase_frameset. $\mathrm{htm}$. Acceso 22/06/09.

Helversen V.O. \& C.C. Voigt. 2002. Glossophagine bat pollination in Helicteres baruensis (Sterculiaceae). Ecotropica 8: 23-30.

Lemke T. O. 1985. Pollen carrying by the nectar-feeding bat Glossophaga soricina in a suburban environment. Biotropica 17(2): 107-111.

Knudsen J.T \& B.B. Klitgaard. 1998. Floral scent and pollination in Browneopsis disepala (Leguminosae: Caesalpinoideae) in western Ecuador. Brittonia 50(2): 174-182.

Lobo J.A., M. Quesada \& K.E. Stoner, et al. 2003. Factors affecting phenological patterns of Bombacaceous trees in seasonal forests in Costa Rica and México. American Journal of Botany 90(7): 1054-1063.

Muchhala N. \& P. Jarrín-V. 2002. Flower visitation by bats in Cloud Forests of western Ecuador. Biotropica 34(3): 387-395.

Pacheco V., R. Cadenillas, S. Velazco, et al. 2007. Noteworthy bat records from the Pacific Tropical rainforest region and adjacent dry forest in northwestern Peru. Acta Chiropterologica 9(2): 409-422.

Pacheco V., R. Cadenillas, E. Salas, et al. 2009. Diversidad y endemismo de los mamíferos del Perú. Revista Peruana de Biología 16(1): 005-032.

Quesada M., K. Stoner., R. Guerrero, et al. 2004. Effects of forest fragmentation on pollinator activity and consequences for plant reproductive success and mating patterns in bat-pollinated Bombacaceous trees. Biotropica 36(2): 131-138.

Quesada M., K. Stoner, R. Guerrero, et al. 2003. Effects of habitat disruption on the activity of nectarivorous bats (Chiroptera: Phyllostomidae) in a dry tropical forest: implications for the reproductive sucess of the Neotropical tree Ceiba grandiflora. Oecología 135: 400-406.

Roubik D. \& J. Moreno. 1991. Pollen and spores of Barro Colorado Island. Volumen 36. Missouri Botanical Garden, Panamá. $268 \mathrm{pp}$.

Sáenz C. \& H. Blume. 1978. Introducción a la Palinología y vocabulario palinológico. 1ra ed., Ediciones Rosario, Madrid, España. $150 \mathrm{pp}$.

Sahley C. 1996. Bat and hummingbird pollination of an autotetraploid columnar cactus, Weberbauerocereus weberbaueri (Cactaceae). America Journal of Botany 83(10): 13291336.

Sánchez N. \& T. Álvarez. 2000. Palinofagia de los murciélagos del género Glossophaga (Mammalia: Chiroptera) en México. Acta Zool. Mex. (n.s) 81: 23-62.

Sazima M., S. Buzato \& I. Sazima. 1999. Bat-pollinated flower assemblages and bat visitors at two Atlantic Forest sites in Brazil. Annals of Botany 83: 705-712.

Simmons N.B. \& A.L. Wetterer. 2002. Phylogeny and Convergence in Cactophilic Bats. In: Fleming T. H \& Valiente-Banuet, eds. Columnar cacti and their mutualists: Evolution, ecology, and conservation. Chapter 5. The University of Arizona Press, Tucson. Pp. 87-93. 